\title{
Estado nutricional e consumo alimentar de pré-escolares e escolares de escola privada
}

\author{
Nutritional status and food intake of preschoolers and school children from a private school
}

\author{
Mônica Glória Neumann Spinelli ${ }^{1}$, Juliana Masami Morimoto ${ }^{2}$, Ana Paula Gumieri de \\ Freitas $^{3}$, Camila Machado de Barros ${ }^{3}$, Débora Helena Silveira Dias ${ }^{3}$, Marina Brosso \\ Pioltine $^{3}$, Patrícia Pacheco de Oliveira Gonçalves ${ }^{3}$, Renata Basaglia Navarro ${ }^{3}$
}

\begin{abstract}
RESUMO
Objetivo: Avaliar o estado nutricional e a adequação do consumo alimentar em pré-escolares e escolares de uma escola privada.

Materiais e Métodos: Estudo transversal realizado com 216 crianças de uma escola privada localizada na região central da cidade de São Paulo, Brasil. Foram aferidos peso e altura e a partir disso calculado o índice de massa corporal, que foi analisado a partir das curvas de crescimento da Organização Mundial da Saúde. Foi solicitado aos pais o preenchimento de um questionário de frequência alimentar para crianças.

Resultados: A prevalência de desnutrição foi de $0,9 \%$, sobrepeso $22,1 \%$ e obesidade $18,9 \%$. Houve inadequação de consumo em todos os grupos com exceção do grupo alimentar de óleos e gorduras. Requerem especial atenção os grupos de hortaliças, frutas, carnes, ovos e doces. O maior consumo de hortaliças teve uma associação estatisticamente significativa com o baixo índice de massa corporal.

Conclusão: São altos os índices de sobrepeso e obesidade na população estudada, além da inadequação de consumo, evidenciando a necessidade de ações educativas para essa população.
\end{abstract}

Palavras-chave: estado nutricional; obesidade; criança; pré-escolar; consumo alimentar.

\begin{abstract}
Objective: To evaluate the nutritional status and dietary intake adequacy of preschool and school children from a private school.

Materials and Methods: Cross-sectional study with 216 children from a private school located in the central region of the city of Sao Paulo, Brazil. Weight and height have been measured and the body mass index has been calculated. Data have been analyzed under the World Health Organization Child Growth Standards. Parents have been requested to fill out a food frequency questionnaire for children.

Results: The prevalence of malnutrition was $0.9 \%$, overweight $22.1 \%$ and obesity $18.9 \%$. Almost all the food groups have shown inadequate intake, with the exception of oils and fats. The groups of vegetables, fruits, meats, eggs and sweets require special attention. The higher consumption of fruits and vegetables had a statistically significant association with low body mass index.

Conclusions: The rates of overweight and obesity are high in this population, besides food inadequacy, highlighting the need of educational activities for this population.
\end{abstract}

Keywords: nutritional status; obesity; preschool child; food consumption.

\footnotetext{
${ }^{1}$ Nutricionista. Doutora em Saúde Pública pela Faculdade de Saúde Pública da Universidade de São Paulo (USP), Brasil. Docente do Curso de Nutrição do Centro de Ciências Biológicas e da Saúde, Universidade Presbiteriana Mackenzie, São Paulo, Brasil.

${ }^{2}$ Nutricionista. Doutora em Ciências pela Faculdade de Saúde Pública da USP, Brasil. Docente do Curso de Nutrição do Centro de Ciências Biológicas e da Saúde, Universidade Presbiteriana Mackenzie, São Paulo, Brasil.

${ }^{3}$ Nutricionistas graduadas pela Universidade Presbiteriana Mackenzie, São Paulo, Brasil.
} 


\section{INTRODUÇÃO}

A prevalência de obesidade e sobrepeso não apenas está aumentando mundialmente, como também apresenta o aumento de sua incidência em faixas etárias mais jovens. Tem-se observado a maior prevalência de obesidade em crianças de todas as idades $^{1,2}$. A proporção de pré-escolares, escolares e adolescentes classificados com sobrepeso tem sofrido um grande aumento nos anos de 1990, após uma relativa estabilidade entre as décadas de 1960 e $1970^{1,2}$. Assim, a obesidade se torna difícil de ser tratada e pode ter consequências adversas, tanto físicas como também emocionais e sociais. Crianças com sobrepeso apresentam maiores chances de se tornarem adultos obesos e sabe-se que o escolar que tem um dos pais com obesidade tem cerca de $70 \%$ de chance de se tornar um adulto obeso ${ }^{3}$.

Valores elevados na prevalência de obesidade infantil também foram observados nos países em desenvolvimento. No Brasil, no período entre 1974 e 1997, a prevalência de sobrepeso e obesidade em crianças e adolescentes aumentou de $4,1 \%$ para $13,9 \%$. $\mathrm{Na}$ China, a prevalência de sobrepeso e obesidade entre crianças de seis anos de idade aumentou de $7,7 \%$ para $12,4 \%$ em um curto período de tempo, entre 1991 e 1997, e no Chile, entre 1987 e 2000, neste mesmo grupo etário verificou-se o aumento na prevalência de $12 \%$ para $26 \%$ para os meninos e de $14 \%$ para $27 \%$ em meninas ${ }^{4}$.

Nos países em desenvolvimento as causas deste aumento provavelmente incluem mudanças no estilo de vida, na prática de atividade física e no padrão da dieta que acompanham a urbanização e o desenvolvimento econômico. Atualmente são realizados grandes investimentos para abordar a questão da perda de peso, levando-se em consideração as consequências fisiológicas, sociais e psicológicas acarretadas pelo excesso de peso ${ }^{5}$.

Apesar de estudos indicarem que a alimentação influencia diretamente a saúde, tanto positivamente como negativamente, e que é um dos fatores mais importantes para a longevidade da população com qualidade de vida ${ }^{6}$, a questão da mensuração do consumo alimentar é, ainda, o grande desafio da ciência e da prática da nutrição e dietética devido às limitações dos métodos para medir esta ingestão de forma fidedigna. O questionário de frequência alimentar (QFA) é considerado um dos principais instrumentos utilizados para estudos epidemiológicos, possibilitando estimar o consumo da maioria dos alimentos em um determinado período pregresso de tempo ${ }^{7}$. Esta característica permite estudar a dieta habitual, que é de fundamental importância para estimar a medida de exposição aos fatores dietéticos e sua influência na qualidade de vida da população ${ }^{7}$.

Estudos epidemiológicos mostram que o sobrepeso na infância está associado ao sobrepeso em adultos, e estudos indicam que a obesidade na infância aumenta de 50 a $80 \%$ a taxa de mortalidade do adulto ${ }^{8}$. Por este motivo e pelo aumento dos riscos de morbidades associadas ao excesso de adiposidade corporal, o estudo do excesso de peso em crianças e adolescentes está se tornando uma preocupação de saúde pública ${ }^{9,10}$. As intervenções para a prevenção da obesidade devem ser iniciadas precocemente, na idade pré-escolar ou mesmo após o nascimento ${ }^{11,12}$. As crianças constituem, portanto, um dos principais grupos-alvo para estratégias de prevenção e controle do sobrepeso e da obesidade, não só devido às suas características como grupo de risco, mas também devido às possibilidades de efetividade das ações preventivas, sendo o ambiente escolar bastante atrativo para investigar a ocorrência de excesso de peso entre as crianças, pois facilita 0 contato com as famílias e possibilita o desenvolvimento de programas de intervenção e controle do sobrepeso infantil através dos conhecimentos que podem ser obtidos desde a infância ${ }^{4}$.

Nesse contexto, o presente estudo teve como objetivo avaliar o estado nutricional e a adequação da dieta por meio de um questionário de frequência alimentar em crianças de uma escola privada na cidade de São Paulo, Brasil, a fim de verificar a prevalência de excesso de peso infantil e sua relação com o consumo alimentar.

\section{MATERIAIS E MÉTODOS}

Trata-se de um estudo transversal, desenvolvido com crianças de uma escola privada da cidade de São Paulo no período de janeiro a novembro de 2009, com uma 
TABELA 1 - Distribuição em número e percentual de crianças segundo altura para idade e sexo.

\begin{tabular}{lcccc}
\hline Classificação de altura para idade & Sexo feminino $\mathbf{n}(\%)$ & Sexo masculino $\mathbf{n}(\%)$ & Total $\mathbf{n}(\%)$ & Valor $\mathbf{p}^{*}$ \\
\hline Baixa estatura & $3(2,9)$ & $3(2,7)$ & $6(2,8)$ & 0,945 \\
Estatura adequada & $102(97,1)$ & $108(97,3)$ & $210(97,2)$ \\
Total & $105(100)$ & $111(100)$ & $216(100)$ & \\
\hline${ }^{*}$ teste do qui-quadrado para diferença de proporções segundo sexo.
\end{tabular}

amostra de 216 crianças menores de dez anos de idade e de ambos os sexos. Foram convidadas a participar todas as crianças menores de dez anos e o critério de exclusão foi a recusa do responsável ou o não preenchimento do questionário de frequência alimentar. A escola foi escolhida por conveniência, uma vez que concordou em participar da pesquisa, mas apresenta a peculiaridade de ter alunos de diversas regiões de São Paulo, apesar de estar situada na região central do município.

Antes do início da pesquisa, esta foi apresentada à escola. Os pais e responsáveis pelas crianças receberam uma breve explicação sobre a pesquisa em uma reunião realizada na escola, na qual se esclareceu que a participação das crianças era voluntária, sem prejuízo daquelas que não fossem participar, e também foi explicado que toda informação coletada seria importante para o diagnóstico nutricional que seria realizado. Os pais que permitissem a participação da criança na pesquisa deveriam assinar e devolver o termo de consentimento livre e esclarecido.

A pesquisa foi submetida para análise e aprovada pelo Comitê de Ética em Pesquisa da Universidade Presbiteriana Mackenzie, São Paulo, Brasil, sob processo CEP/UPM $n^{0} 1108 / 02 / 2009$ e CAAE $n^{\circ}$ 0003.0.272.000=09, obedecendo a todos os preceitos da Resolução 196/96 do Conselho Nacional de Saúde em todas as fases de sua execução.

A avaliação do estado nutricional foi feita a partir da aferição do peso e estatura para o posterior cálculo do índice de massa corporal (IMC). Para a obtenção do peso corporal, foram utilizadas duas balanças antropométricas digitais portáteis da marca $G$ Tech $\AA$, com capacidade de 150 quilos e precisão de 100 gramas. Os escolares foram avaliados conforme procedimentos recomendados pelo Ministério da Saúde $^{13}$. Para a obtenção da estatura dos escolares utilizou-se estadiômetro de 2 metros, com precisão de 0,1 centímetro. Foi solicitado que a criança ficasse em pé, descalça, com os calcanhares, panturrilhas, glúteos e ombros encostados à parede e a cabeça posicionada com o plano de Frankfurt.

Os dados de peso e altura foram convertidos em índices antropométricos de peso para idade, altura para idade e IMC para idade em z-escore pelas curvas de crescimento da Organização Mundial da Saúde ${ }^{14}$. Para o cálculo dos índices antropométricos em z-escore foi utilizado o Programa WHO Anthro (versão 3.1, World Health Organization, Geneva, 2009).

A caracterização do consumo alimentar foi feita por meio do questionário de frequência alimentar validado (QFA) para crianças ${ }^{7}$, já que é um dos métodos mais utilizados por ter a vantagem de medir o consumo alimentar habitual, além de ser de fácil utilização e de apresentar baixo custo. O QFA utilizado possui 48 alimentos e teve sua validade e reprodutibilidade testada em amostra de crianças de 2 a 5 anos de idade. Os detalhes sobre a construção do QFA estão descritos na literatura ${ }^{7}$. O QFA foi entregue aos pais e responsáveis das crianças para que estes fossem preenchidos e devolvidos para a escola, no prazo de uma semana, juntamente com a carta de informação ao sujeito da pesquisa e o termo de consentimento livre e esclarecido.

O consumo em número de porções de cada grupo alimentar foi comparado às recomendações para cada faixa etária da infância ${ }^{15,16}$, sendo estas utilizadas pela Sociedade Brasileira de Pediatria para orientações sobre a alimentação do pré-escolar e do escolar ${ }^{17}$.

As análises estatísticas foram realizadas com 0 software STATA, versão 7. A análise da proporção de crianças segundo categorias das variáveis qualitativas foi realizada por meio do teste do qui-quadrado, e a análise da média de uma variável quantitativa segundo 
TABELA 2 - Distribuição em número e percentual de crianças segundo classificação do estado nutricional por IMC para idade.

\begin{tabular}{|c|c|c|c|c|}
\hline Classificação do IMC para idade & \multicolumn{2}{|c|}{ Faixa etária (anos) - n (\%) } & Total n (\%) & Valor $p$ \\
\hline Baixo IMC para idade & --- & $2(1,6)$ & $2(0,9)$ & $0,209^{*}$ \\
\hline Eutrofia & $57(64,0)$ & $65(51,2)$ & $122(56,5)$ & \\
\hline Sobrepeso & $18(20,2)$ & $35(27,5)$ & $53(24,5)$ & \\
\hline Obesidade & $14(15,7)$ & $25(19,7)$ & $39(18,1)$ & \\
\hline Total & $89(100)$ & $127(100)$ & $216(100)$ & \\
\hline
\end{tabular}

${ }^{*}$ teste do Qui-quadrado para diferença de proporções segundo idade.

três ou mais categorias de uma variável qualitativa foi realizada através da análise de variância (ANOVA). Este estudo levou em consideração valores significativos como sendo $p \leq 0,05$.

\section{RESULTADOS}

Dos 216 participantes, 111 (51,4\%) eram do sexo masculino. Em relação à idade 89 eram menores de sete anos e 127 tinham sete anos e mais. Na amostra pesquisada $39,8 \%$ das crianças eram moradoras da região centro, $24 \%$ provenientes da zona Oeste, $10 \%$ da zona Leste, $12,5 \%$ da zona Sul, $10,3 \%$ da zona Norte e $3,4 \%$ da região metropolitana de São Paulo (Caieiras, Barueri, Guarulhos, Suzano, Ferraz de Vasconcelos, ABCD). Com relação ao nível socioeconômico, 19\% das crianças da amostra eram de famílias com renda familiar inferior a 1,5 salário mínimo, devido ao programa de bolsa concedido pela escola.

Salienta-se que o presente estudo apresentou uma taxa de não-resposta de 57,6\% em decorrência da não participação ou recusa por parte dos familiares das crianças. A perda na amostra por falta de algum dos dados representou $3,13 \%$.

Não foi observada diferença estatisticamente significativa entre altura para a idade e sexo como pode ser observado na tabela 1. Em ambos os sexos verificou-se que a maior parte das crianças apresentava altura adequada para a idade. A tabela 2 apresenta os resultados de avaliação do IMC para idade, que demonstrou alto percentual de sobrepeso e de obesidade no grupo (42,6\%). Embora encontrado um percentual de sobrepeso e obesidade maior na faixa etária de crianças de sete anos e mais, não foi observada diferença estatisticamente significativa.
Utilizando-se o índice de massa corporal para idade e sexo (tabela 3) foi possível observar que mais de $40 \%$ das crianças avaliadas apresentaram sobrepeso ou obesidade. Foi observada uma maior porcentagem de crianças com sobrepeso e obesidade entre os meninos, mas sem significância estatística.

Em relação ao consumo alimentar, observou-se na tabela 4 um grande percentual de consumo inadequado de praticamente todos os grupos de alimentos, o que pode ocasionar grandes prejuízos na ingestão de nutrientes e, consequentemente, na saúde das crianças.

Neste estudo, em alguns grupos de alimentos como hortaliças, frutas, carnes, ovos, doces e açúcares, a porcentagem de crianças que não apresentaram 0 consumo adequado foi superior ao das crianças que apresentaram adequação. Verifica-se a necessidade de atenção em relação aos grupos de cereais, leguminosas, leite e derivados, cuja inadequação atingiu mais de um terço da população estudada. Com relação às gorduras, que alcançaram um bom nível de adequação, é importante que se desenvolvam novos estudos para identificar possíveis falhas do instrumento de coleta de dados. Também foi verificado que o consumo de verduras, legumes e frutas - alimentos fontes de vitaminas e minerais - foi considerado inadequado, sugerindo que a ingestão dos nutrientes presentes nestes alimentos possa, possivelmente, ser deficiente.

A tabela 5 apresenta a comparação da média de consumo dos grupos de alimentos segundo categorias do IMC para idade. Ao analisar a média do consumo do número de porções segundo o estado nutricional das crianças, avaliado pelo índice de massa corporal para idade, apenas o consumo médio de hortaliças 
TABELA 3 - Distribuição em número e percentual de crianças segundo classificação do estado nutricional por IMC para idade e sexo.

\begin{tabular}{|c|c|c|c|c|}
\hline Classificação do IMC para idade & Sexo feminino $\mathrm{n}(\%)$ & Sexo masculino $\mathrm{n}(\%)$ & Total $\mathbf{n}(\%)$ & Valor $p$ \\
\hline Baixo IMC & $1(1,0)$ & $1(0,9)$ & $2(0,9)$ & 0,772 \\
\hline Eutrofia & $63(60,0)$ & $59(53,2)$ & $122(56,5)$ & \\
\hline Sobrepeso & $23(21,9)$ & $30(27,0)$ & $53(24,5)$ & \\
\hline Obesidade & $18(17,1)$ & $21(18,9)$ & $39(18,1)$ & \\
\hline Total & $105(100)$ & $111(100)$ & $216(100)$ & \\
\hline
\end{tabular}

*teste do qui-quadrado para diferença de proporções segundo sexo.

apresentou diferença estatisticamente significativa, mostrando tendências de redução no consumo de verduras e legumes conforme aumenta o IMC.

\section{DISCUSSÃO}

Com relação ao número total de crianças avaliado, consequente a alta taxa de não resposta, acredita-se que a grande perda se deva à aplicação do QFA, que demanda um período de tempo razoável para o preenchimento por parte dos pais ou responsáveis. Esse percentual de devolução acaba sendo um fator limitante do estudo, pois além de restringir o tamanho da amostra, pode encobrir uma inadequação de consumo ou de estado nutricional, uma vez que não é possível saber se os não respondentes 0 fizeram intencionalmente para não evidenciar uma situação de inadequação.

A adequação da altura para a idade neste estudo é semelhante à encontrada por Pazin et al. ${ }^{18}$ em pesquisa com pré-escolares e escolares. Essa condição possivelmente evidencie uma melhor qualidade de vida e de alimentação dessas crianças de escola privada e de melhor nível sócio-econômico. Segundo Onis et al. ${ }^{19}$, o crescimento, além de ser o melhor indicador global de bem-estar infantil, é também um excelente indicador de desigualdades nas populações. Provavelmente este contexto seja também responsável pelo alto índice de sobrepeso e obesidade encontrado na população deste estudo (42,6\%), superior ao encontrado por Pazin et al. ${ }^{18}$. O aumento considerável do número de crianças com obesidade é considerado preocupante, pois nessa fase da vida ocorre o desenvolvimento das células adiposas - fator determinante para os padrões de composição corporal do indivíduo adulto ${ }^{20}$.
O índice de massa corporal para idade se mostra o índice antropométrico mais apropriado para a realização do diagnóstico nutricional de crianças por levar em consideração as medidas de peso, altura e idade conforme o proposto pela Organização Mundial da Saúde ${ }^{14}$ e, portanto, levou-se em consideração este índice antropométrico como sendo o mais adequado para o cálculo da prevalência de crianças com sobrepeso e obesidade no presente estudo. Nos últimos 30 anos, o número de crianças com peso acima do adequado triplicou no Brasil, enquanto nos Estados Unidos os valores duplicaram. Esse aumento foi mais importante nas regiões sul e sudeste do Brasil, onde se verifica uma melhor situação econômica ${ }^{21}$.

No presente estudo verificou-se a alta porcentagem de crianças que não apresentaram o consumo adequado nos grupos de cereais e de leguminosas, os quais são alimentos de alta disponibilidade e de baixo custo. Esse resultado confirma recentes pesquisas que mostram a diminuição do consumo desses grupos de alimentos, que sempre fizeram parte do hábito alimentar brasileiro e em especial das crianças. A combinação de arroz e feijão é benéfica ao organismo principalmente quanto ao fornecimento de aminoácidos essenciais ${ }^{22}$.

Quanto ao consumo alimentar, este tem sido relacionado à obesidade não somente pelo volume da ingestão alimentar, como também pela qualidade dos alimentos presentes na dieta ${ }^{23}$. O consumo inadequado de frutas, hortaliças e leite, associado ao aumento no consumo de guloseimas, como bolachas recheadas, salgadinhos, doces e refrigerantes contribui com 0 aumento da prevalência de excesso de peso em crianças $^{24}$.

No presente estudo o consumo adequado de hortaliças mostrou-se significativo aos menores valores 
TABELA 4 - Distribuição em número e porcentagem de crianças segundo a adequação de consumo dos grupos de alimentos.

\begin{tabular}{lcc}
\hline Grupo de alimentos & Consumo inadequado $\mathbf{n}(\%)$ & Consumo adequado $\mathbf{n}(\%)$ \\
\hline Cereais, massas, tubérculos e raízes & $82(36,8)$ & $141(63,2)$ \\
Leguminosas & $107(48,0)$ & $116(52,0)$ \\
Hortaliças & $200(89,7)$ & $23(10,3)$ \\
Frutas & $161(72,2)$ & $62(27,8)$ \\
Carnes e ovos & $150(67,3)$ & $73(32,7)$ \\
Leite e derivados & $107(48,0)$ & $116(52,0)$ \\
Doces e açúcares & $151(67,7)$ & $72(32,3)$ \\
Óleos e gorduras & $18(8,1)$ & $205(91,9)$ \\
\hline
\end{tabular}

de índice de massa corporal nas crianças estudadas. As hortaliças e frutas são alimentos considerados como importantes fontes de vitaminas e fibras, sendo assim recomendadas para uma dieta equilibrada ${ }^{25}$. Assim como neste estudo, na pesquisa de Fagundes et al. ${ }^{26}$ os portadores de sobrepeso e os obesos consumiam menos verduras e legumes. A elevada inadequação do consumo de carnes e ovos, verificada no presente estudo, indica a necessidade de avaliação de parâmetros bioquímicos indicadores de anemia ferropriva. As crianças normalmente constituem um grupo de risco para a deficiência de ferro, o que em associação a uma alimentação pobre em alimentos fontes deste nutriente pode acarretar no desenvolvimento de anemia ferropriva ${ }^{27,28}$.

No que se refere ao grupo dos alimentos lácteos, grande percentual das crianças apresentou o consumo insuficiente de leite e derivados, o que se torna preocupante considerando que os laticínios são a melhor fonte de cálcio da alimentação. O cálcio é um elemento fundamental ao organismo, e sua importância está relacionada às funções que desempenha na mineralização óssea, desde a formação, até a manutenção da estrutura e rigidez do tecido ósseo. Sabe-se também que a infância e a adolescência são períodos de maior aumento da massa óssea, e que a ingestão inadequada de cálcio pode prejudicar então o crescimento e desenvolvimento ósseo nas crianças, além de predispor os escolares ao desenvolvimento de osteomalácia ou osteoporose na idade adulta ${ }^{29}$.

No presente estudo foi observada a elevada participação de alimentos com baixo valor nutricional como os doces e alimentos com alto teor de açúcares simples, o que pode trazer consequências negativas à saúde. Observou-se que $67,7 \%$ das crianças tinham consumo inadequado de açúcares e doces. Resultados semelhantes ocorrem no país, onde se verificou o consumo excessivo de alimentos com alto teor de açúcares e o baixo consumo de frutas e hortaliças ${ }^{20}$. Assim, o padrão alimentar brasileiro tem apresentado mudanças decorrentes do maior consumo de alimentos industrializados em substituição às tradicionais comidas caseiras e alimentos in natura. Estas transformações provocadas pelo estilo de vida moderno levam ao consumo excessivo de produtos gordurosos, açúcares, doces e à diminuição da ingestão de cereais, produtos integrais, frutas e verduras, os quais são fontes de fibras, vitaminas e minerais ${ }^{22,25}$. Em estudo realizado com 213 crianças de uma escola privada da cidade de São Paulo, verificou-se que $60,6 \%$ das crianças relataram não ter o hábito de consumir frutas no café da manhã, fato este que também é considerado importante por contribuir para uma menor ingestão de vitaminas e minerais essenciais ao crescimento e desenvolvimento dos escolares ${ }^{30}$.

A partir dos resultados obtidos e das questões expostas conclui-se que são altos os índices de sobrepeso e obesidade na população estudada, pois atingiu 22,1 e $18,9 \%$ do total da amostra, respectivamente, e que as prevalências de sobrepeso e obesidade foram maiores no sexo masculino. Observouse a inadequação de consumo para todos os grupos de alimentos com exceção do grupo alimentar de óleos e gorduras, e que o maior consumo de hortaliças teve 
TABELA 5 - Média do número de porções consumido, segundo grupo de alimentos e classificação do IMC para idade.

\begin{tabular}{llcccc}
\hline \multirow{2}{*}{ Grupo de alimentos } & \multicolumn{3}{c}{ Média do número de porções \pm desvio padrão } & \multirow{2}{*}{ Valor $\mathbf{p}^{*}$} \\
\hline Cereais, massas, tubérculos e raízes & Baixo IMC & Eutrofia & Sobrepeso & Obesidade & \\
Leguminosas & $9,0 \pm 3,0$ & $7,1 \pm 2,1$ & $6,6 \pm 2,0$ & $6,9 \pm 2,0$ & 0,2851 \\
Hortaliças & $1,0 \pm 0,0$ & $0,9 \pm 0,7$ & $1,0 \pm 0,7$ & $0,9 \pm 0,7$ & 0,9519 \\
Frutas & $4,2 \pm 1,1$ & $1,7 \pm 1,4$ & $1,8 \pm 1,5$ & $1,3 \pm 1,3$ & 0,0259 \\
Carnes e ovos & $1,4 \pm 0,4$ & $2,8 \pm 2,0$ & $2,9 \pm 1,9$ & $2,6 \pm 1,8$ & 0,6919 \\
Leite e derivados & $1,6 \pm 0,2$ & $1,9 \pm 1,1$ & $2,2 \pm 2,0$ & $1,8 \pm 1,2$ & 0,5674 \\
Açúcares e doces & $3,1 \pm 0,3$ & $3,1 \pm 1,6$ & $3,3 \pm 1,8$ & $3,4 \pm 1,7$ & 0,6727 \\
Óleos e gorduras & $3,1 \pm 1,8$ & $2,8 \pm 1,4$ & $3,1 \pm 1,8$ & $2,8 \pm 1,3$ & 0,5880 \\
\hline *análise de varîncia das & $0,7 \pm 0,4$ & $0,6 \pm 0,5$ & $0,7 \pm 0,6$ & $0,7 \pm 0,6$ & 0,8058 \\
\hline
\end{tabular}

*análise de variância das médias segundo categorias do IMC para idade

uma associação estatisticamente significativa com valores menores do índice de massa corporal. Sugerese a prática de atividades de intervenção e orientação nutricional no ambiente estudado visando à promoção da qualidade de vida infantil por meio da alimentação adequada.

\section{Agradecimentos}

Ao Instituto Presbiteriano Mackenzie pelo financiamento da pesquisa. À Profa. Maria Ignez Godoy Giandalia pela coleta e disponibilização dos dados antropométricos.

\section{REFERÊNCIAS}

1. Batista Filho M, Rissin A. A transição nutricional no Brasil: tendências regionais e temporais. Cad Saúde Pública. 2003;19(Suppl 1):S181-91.

2. Lobstein $T$, Baur L, Uauy R. Obesity in children and young people: a crisis in public health. Obes Rev. 2004; 5(Suppl 1):S4-85.

3. Dietz WH. Health consequences of obesity in youth: childhood predictors of adult disease. Pediatrics. 1998; 101(1):518-25.

4. Wang Y, Monteiro C, Popkin BM. Trends of obesity and underweight in older children and adolescents in the United States, Brazil, China, and Russia. Am J Clin Nutr. 2002; 75(6):971-7.

5. Cagliari MP, Paiva AA, Queiroz D, Araujo ES. Consumo alimentar, antropometria e morbidade em pré-escolares de creches públicas de Campina Grande, Paraíba. Rev Soc Bras Alim Nutr. 2009; 34(1):29-43.

6. Willett WC. Nutritional epidemiology. $2^{\mathrm{a}}$. ed. New York: Oxford University Press; 1998.

7. Colucci ACA, Philippi ST, Slater B. Desenvolvimento de um questionário de frequência alimentar para avaliação do consumo alimentar de crianças de 2 a 5 anos de idade. Rev Bras Epidemiol. 2004;7(4):393-401.

8. Troiano RP, Briefel RR, Carroll MD, Bialostosky K. Energy and fat intakes of children and adolescents in the United states: data from the National Health and Nutrition Examination Surveys. Am J Clin Nutr. 2000; 72(Suppl 1): 1343S-53S.
9. Damiani D, Carvalho DP, Oliveira RG. Obesidade na infância - um grande desafio. Pediatria Moderna. 2000; 36(8): 489-528.

10. Ministério da Saúde. Política nacional de demografia e saúde. Saúde e estado nutricional de crianças menores de 5 anos. Brasília (DF): Ministério da Saúde; 2006.

11. Moreno LA, Rodríguez G. Dietary risk factors for development of childhood obesity. Curr Opin Clin Nutr Metab Care. 2007;10(3):336-41.

12. Fagundes $A A$, Barros DC, Duar HA. Vigilância alimentar e nutricional: orientações básicas para a coleta, o processamento, análise de dados e a informação em serviços de saúde. Brasília (DF): Ministério da Saúde; 2004.

13. Ministério da Saúde. Sisvan: orientações básicas para a coleta, processamento, análise de dados e informação em serviços de saúde. Brasília (DF): Ministério da Saúde; 2004. Série A. Normas e Manuais Técnicos.

14. World Health Organization. Growth reference data for 5-19 years, 2007. [internet]. [citado 2009 jan 02]; Disponível em: http://www.who.int/growthref/en/.

15. Monteiro CA, Conde WL. Tendência secular da desnutrição e da obesidade na infância na cidade de São Paulo (1974-1996). Rev Saúde Pública. 2000;34(Supl 6): 52-61.

16. Vitolo MR, Gama CM, Bortolini GA, Campagnolo PD, Drachler ML. Some risk factors associated with overweight, stunting and wasting among children under 5 years old. J Pediatr. 2008; 84(3):251-7.

17. Sociedade Brasileira de Pediatria. Manual de orientação: alimentação do lactente, alimentação do pré-escolar, alimentação do escolar, alimentação do adolescente, alimentação na escola. São Paulo (SP): Departamento de Nutrologia; 2006.

18. Pazin JR, Donadone VS, Abreu ES, Simony RF. Prevalência de sobrepeso e obesidade em pré-escolares e escolares de escolas particulares. Rev Ciência \& Saúde. 2012;5(2):87-91.

19. Onis $M$ de, Frongillo EA, Blossner $M$. Is malnutrition declining? An analysis of changes in levels of child malnutrition since 1980. Bull World Health Organ. 2000; 78(10):1222-33.

20. Triches RM, Giugliani ERJ. Obesidade, práticas alimentares e conhecimentos de nutrição em escolares. Rev Saúde Pública. 2005;39(4):541-7.

21. Kosti RI, Panagiotakos DB. The epidemic of obesity in children and adolescents in the world. Cent Eur J Public Health. 2006;14(4):151-9. 
22. Resende MA, Pereira DA, Marins SS. Cuidados na alimentação de crianças em instituições de educação infantil. Fam Saúde Desenvol. 2007; 8(1):32-41.

23. Philippi ST, Colucci ACA, Cruz ATR, Ferreira MN, Coutinho RLR. Alimentação saudável na infância e na adolescência. In: Curso de atualização em alimentação e nutrição para professores da rede pública de ensino, 2000; Piracibaca. Piracicaba: Escola Superior de Agricultura Luiz de Queiroz; 2000. p.46-60.

24. Zlochevsky ELR. Obesidade na infância e adolescência. Rev Paul Pedriatria. 1996;14(3):124-32.

25. Dietz WH, Gortmaker SL. Preventing obesity in children and adolescents. Annu Rev Public Health. 2001;22(1): 337-53.

26. Fagundes ALN, Ribeiro DC, Naspitz L, Garbelini LEB, Vieira JKP, Silva AP, Lima VO, Fagundes DJ, Compri PC, Juliano Y. Prevalência de sobrepeso e obesidade em escolares da região de Parelheiros do município de São Paulo. Rev Paul Pediatr. 2008;26(3):212-7.

27. Carvalho MC, Baracat ECE, Sgarbieri VC. Anemia ferropriva e anemia de doença crônica: distúrbios do metabolismo de ferro. Seg Alim Nutr. 2006;13(2):54-63.

28. Spinelli MGN. Concentração de hemoglobina, prevalência de anemia e fatores de risco associados, em crianças brasileiras menores de um ano: um estudo multicêntrico de abrangência nacional [tese]. São Paulo (SP): Faculdade de Saúde Pública da USP; 2004.

29. Cobayashi F. Cálcio: seu papel na nutrição e saúde. Compacta Nutr. 2004; 5(2):3-18.

30. Pioltine MB, Spinelli MGN. Conhecimentos sobre nutrição e sua relação com o IMC de escolares. Rev Simbio-logias. 2010; 3(4):57-74.

\section{Endereço para correspondência:}

Marina Pioltine

Rua Barão de São Gabriel n 389

São Paulo/SP - CEP 05085-060

Telefone: +55 11999543343

E-mail: marinapioltine@usp.br 